fournal of Medical Genetics (1971). 8, 312.

\title{
Familial Total Anomalous Pulmonary Venous Return
}

\author{
J. E. PAZ and E. E. CASTILLA \\ From the Medical Genetics Centre, Combate de los Pozos 2193, Buenos Aires, Argentina
}

Total anomalous pulmonary venous return is a rare congenital cardiovascular anomaly causing death within the first few months of life.

The cases presented here, are to the authors' knowledge the first reports of the familial recurrence of this anomaly to be published.

\section{Case Reports}

Three patients affected with total anomalous pulmonary venous return were detected within one single family (Fig.). A detailed clinical picture of these patients will be published elsewhere.

mother was 24 and his father 26 years old, and both of them were healthy, at the time of birth; birth weight was $2800 \mathrm{~g}$. The first abnormal signs were pallor and weak suction at the age of 15 days. At 30 days of age the baby presented dyspnoea while feeding, tachycardia, and a chest deformity with an increased anteroposterior diameter. He developed an uncompensated cardiac failure and died at the age of 60 days. Cyanosis was never observed. Necropsy revealed that the 4 pulmonary veins emptied into a common sac which continued through the right azygos, skirting the trachea by its right border, and ending as usual in the superior vena cava. No other congenital malformations were diagnosed.

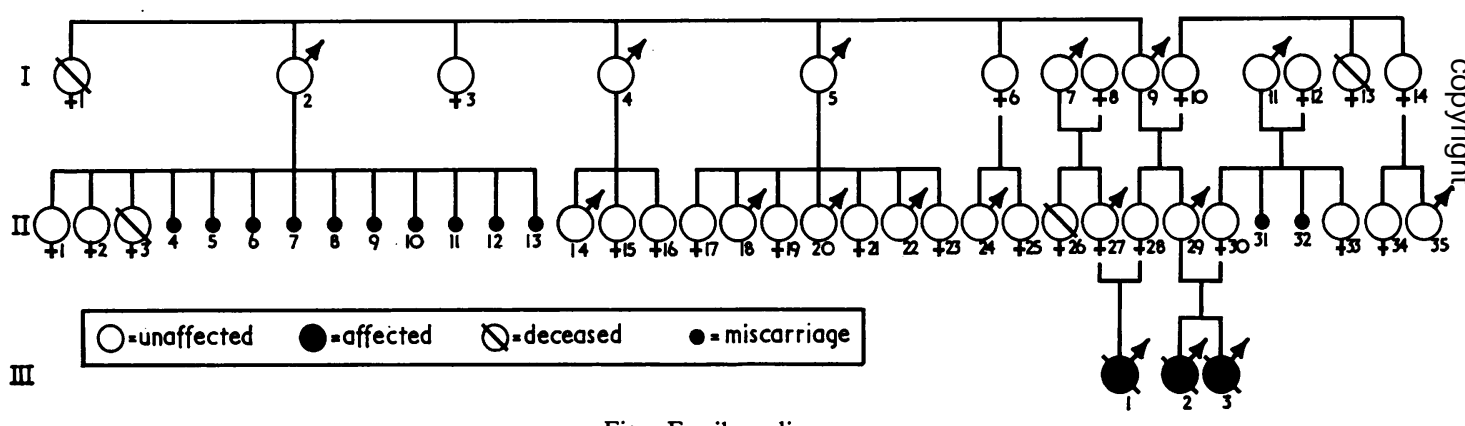

Fig. Family pedigree

Case 1 (III.1). The index case is a male born on 17 July 1969 of healthy parents. At his birth his mother was 25 and his father 28 ; birth weight was $4000 \mathrm{~g}$. A cyanotic congenital heart disease was clinically diagnosed during the first day of life. Cardiac catheterism, at the age of 26 days, led to the diagnosis of total anomalous pulmonary return into the coronary sinus, inferior vena cava or right auricle. He died at 27 days after having been on continuous tracheal intubation for 12 days because of repeated apnoeic crisis. Necropsy revealed that the 4 pulmonary veins emptied into a common sac which communicated with the vena porta by means of a persistent posterior cardial vein. No other congenital anomalies were detected by clinical and pathological examination.

Case 2 (III.2) is a male baby born on 21 June 1968 (13 months before the birth of his first cousin, case 1). His

Received 27 November 197 J.
Case 3 (III.3). This male child was born on 13 June 1969, 12 months after his brother (case 2) and one month before his first cousin (case 1); birth weight was $3500 \mathrm{~g}$. Slight jaundice was present in the first week of life; birth weight was regained at the age of 30 days. No abnormal signs were noticed until age 15 days when tachycardia was observed. Dyspnoea was evident, starting on the 12th day. Cardiac murmur, cardiomegaly, thoracic deformity, and hepatomegaly were present at one month of age. Cardiac catheterism confirmed supracardiac total anomalous pulmonary venous return. A closed atriotomy was then performed by Rashkind's balloon. Open heart surgery was performed at one year because of rapid deterioration in the child's condition; a total anomalous pulmonary venous return of the same type described in case 2 was observed. The patient died one day after the operation. His cardiovascular malformation was the only anomaly to be diagnosed. 


\section{The Family}

The three affected babies were born to two healthy sibs married to unrelated spouses. Consanguinity was not detected within this family. The surnames were different, and the three lines linked to both couples producing total anomalous pulmonary venous return came of mixed Spanish and Italian ancestry. This SpanishItalian mixture is frequently found in the population of Buenos Aires; in addition all their ancestors came from different areas of Spain and Italy.

The mother of case 1 (II.28) and the father of cases 2 and 3 (II.29) were subjected to special cardiovascular examination in order to detect undiagnosed anomalies in both instances the results were normal.

No other cases of congenital heart disease had been detected in this family. There were two individuals who died within the first day of life (II.3 and II.26), but no documented evidence as to the cause of death could be found. The 10 first-trimester spontaneous abortions (from II.4 to II.13) were attributed to a uterus didelphus in the wife of I.2.

\section{Cytogenetic Studies}

Mitotic chromosome studies were performed on cultured peripheral blood leucocytes from case 3 (III.3), from the father of case 3 (II.29), and from the mother of case 1 (II.28). Particular care was taken to detect any minimal structural chromosome aberration. The results were within normal limits for the three subjects investigated.

\section{Discussion}

Total anomalous pulmonary venous return is known to be one of the less common congenital cardiovascular malformations. To date, no reliable data are available on its actual incidence, but it is known to account for about $2 \%$ of all necropsied congenital heart diseased patients (Darling, Rothney, and Craig, 1957).

No published reports on familial recurrence of total anomalous pulmonary venous return have been found in the literature. An instance of a father and daughter both affected with a partial anomalous pulmonary venous return and lung hypoplasia was published by Neill et al (1960). This is an anatomical entity different from total anomalous pulmonary venous return. Nevertheless, the presence of a common aetiopathogenic mechanism cannot be discarded. In an unpublished observation by F. C. Fraser, two sibs, a boy and a girl, out of a sibship of four affected by total anomalous pulmonary venous return are described; the sibship included another female who died from congenital tracheoesophageal fistula and a normal female. The parents were not related, and necropsy confirmed the diagnosis of supracardiac total anomalous pulmonary venous return, in both babies.

The three related cases of total anomalous pulmonary venous return reported here can reasonably be assumed, on the basis of the low frequency of this anomaly, to represent the same aetiological entity in spite of the infracardiac type found in case 1 and of the less severe and less rare (Gott et al, 1956; Keith, Rowe, and Vlad, 1958) supracardiac type in cases 2 and 3.

The observed pedigree distribution of the three affected cases reported here makes it difficult to accept any of the simple genetic or environmental mechanisms usually assumed to explain familial recurrence.

Random recurrence can be disregarded on account of the low incidence of this anomaly in the general population.

A prenatal environmental agent present in three separated pregnancies observed in two unrelated mothers, living in different houses and sharing nothing more than would any other two inhabitants of a large urban area, seems quite unlikely. In addition, if a comparison with previous years is made no change can be observed in the incidence of total anomalous pulmonary venous return in this city in the period under investigation.

Among simple genetic mechanisms, an autosomal recessive mechanism would imply the mating of two heterozygous sibs with two similarly heterozygous unrelated individuals taken at random from the general population. Such a situation could be considered extremely improbable in spite of the lack of real estimates of gene frequency for conditions as rare as these.

Both $\mathrm{X}$ - and $\mathrm{Y}$-linked inheritance are easily discarded because it is necessary to assume two instances of direct male-to-male (II.29, III.2, and III.3) and one instance of female-male (II.28, III.1) transmissions.

A polygenic mechanism seems also an unlikely alternative on account of the recurrence in three individuals within two sibships in the same generation, the fixed phenotypic expressivity, and the low incidence of this anomaly in the general population.

A chromosomal aberration has to be taken into consideration since the pedigree is compatible with a chromosomal translocation being carried in balance by the two related parents (II.28 and II.29) as well as in one of their progenitors and with the translocation being present in the unbalanced form in the three affected children. Nevertheless, as no other anomalies were associated with the total anomalous pulmonary venous return in the three patients, such a chromosome rearrangement would probably 
involve a very small portion of the genome, and would therefore be unlikely to be detected by the available cytogenetic methods. The normal karyotypes of II.28, II.29, and III. 3 cannot rule out the existence of a small undetectable chromosome translocation being transmitted in this family.

Autosomal dominant inheritance would require some protective mechanism preventing the development of the anomaly in at least three individuals carrying the mutant gene; II.28, II.29, and in one of their parents. Incomplete penetrance is not a likely possibility in the presence of a fixed expressivity of clinical and anatomical picture in the three affected children, and due to the lack of clinical, radiological, and electrocardiographic evidence in the two presumed carrier parents.

The absence of a satisfactory explanation of the observed familial recurrence of total anomalous pulmonary venous return in this family by way of the simple genetic mechanisms led the authors to advance another interpretation considered as the most economic besides the aforementioned. This implies the action of two loci, connected in some way to the embryological development of the pulmonary vessels and related by complementarity (Emerson, Beadle, and Fraser, 1935); the wild genotype being aa,bb. A mutation at the $b$ locus will produce an $\mathrm{a}, \mathrm{Bb}$ genotype which corresponds to the abnormal total anomalous pulmonary venous return phenotype. If we assume a mutation at the $a$ locus capable of supressing the effect from the B gene, being the action of the A mutant gene epistatic over the $b$ locus (Fisher, 1918), and $\mathrm{Aa}, \mathrm{Bb}$ genotype could be proposed for both related parents (II.28 and II.29), where the A mutant protects the development of the anomaly induced by the $B$ mutant gene. This mechanism has already been described in humans for the secretor-non secretor state for the $\mathrm{ABH}$ and Lewis blood group system (Grubb, 1951). If the Aa,bb genotype had no pathological effect this could be present in the ancestors (one of the grandparents-I.9 or I.10) a fresh mutant for the B gene which was then inherited without recombination with the A mutant gene to both parents (II.28 and II.29).

The three affected children are all recombinants from their parental genotypes. Thus, the mating of an $\mathrm{Aa}, \mathrm{Bb}$ double heterozygote to a wild homozygote $a a, b b$ from the general population-assuming independent assortment-would lead to a progeny composed of $25 \%$ of healthy $\mathrm{Aa}, \mathrm{Bb}$ double heterozygotes, $25 \%$ of healthy Aa,bb single heterozygotes a, $25 \%$ of healthy aa,bb double wild homozygotes, and $25 \%$ of affected aa, $\mathrm{Bb}$ single heterozygotes $b$.

If this hypothesis is correct many of the cases of total anomalous pulmonary venous return would be fresh mutants for a single gene which is not transmitted to the descendants because of the fatality of this anomaly which avoids reproduction. If so, it would be of interest to collect data on the paternal age of all cases of total anomalous pulmonary venous return and to observe the children produced by those patients who survived open heart surgery as surgical procedures have improved.

\section{Summary}

Three male babies dying from total anomalous pulmonary venous return not associated with other congenital anomalies were found within a single family. Two of them are brothers and the third child is a first cousin, the son of a sister of the father of the first two children. The anomaly was anatomically confirmed in all three patients. The parents were not affected, no consanguinity was detected, and chromosomes studies were normal.

None of the usual genetic mechanisms can satisfactorily explain the observed pedigree distribution. The authors consider the most likely interpretation to be either an undetectable small chromosome translocation, or a single autosomal dominant gene mutation under the epistatic effect of a second? mutant gene at a separated locus protecting the carrier parents from developing the anomaly.

We would like to thank Dr Eduardo Kreutzer and Dr Luis Becú from the Hemodynamics Department and the Pathology Laboratory at the Hospital de Niños de Buenos Aires for their excellent phenotypic description of this family.

\section{REFERENCES}

Darling, R. C., Rothney, W. B., and Craig, J. M. (1957). Total pulmonary venous drainage into the right side of the heart; report of 17 autopsied cases not associated with other major cardiovascular anomalies. Laboratory Investigation, 6, 44.

Emerson, R. A., Beadle, G. W., and Fraser, A. C. (1935). A Summary of Linkage Studies in Maize. Cornell University Agricultural Experiment Station Memoirs, No. 180.

Fisher, R. A. (1918). The correlation between relatives on the supposition of Mendelian inheritance. Transactions of the Royal Society of Edinburgh, 52, 399.

Gott, V. L., Lester, R. G., Lillehei, C. W., and Varaco, R. L. (1956). Total Anomalous Pulmonary Return; an analysis of thirty cases. Circulation, 13, 543.

Grubb, R. (1951). Observations on the human group system Lewis. Acta Pathologica et Microbiologica Scandinavica, 28, 61 .

Keith, J. D., Rowe, R. D., and Vlad, P. (1958). Heart Disease in Infancy and Childhood. Macmillan Co., New York.

Neill, C. A., Ferencz, C., Sabiston, D. C., and Sheldon, H. (1960). The familial occurrence of hypoplastic right lung with systemic arterial supply and venous drainage: 'Scimitar Syndrome'. Bulletin of the fohns Hopkins Hospital, 107, 1-15. 